Antoine CASANOVA, Napoléon et la pensée de son temps : une histoire intellectuelle singulière, Paris, La Boutique de l'histoire, 2000, 324 p.

Claude Mazauric

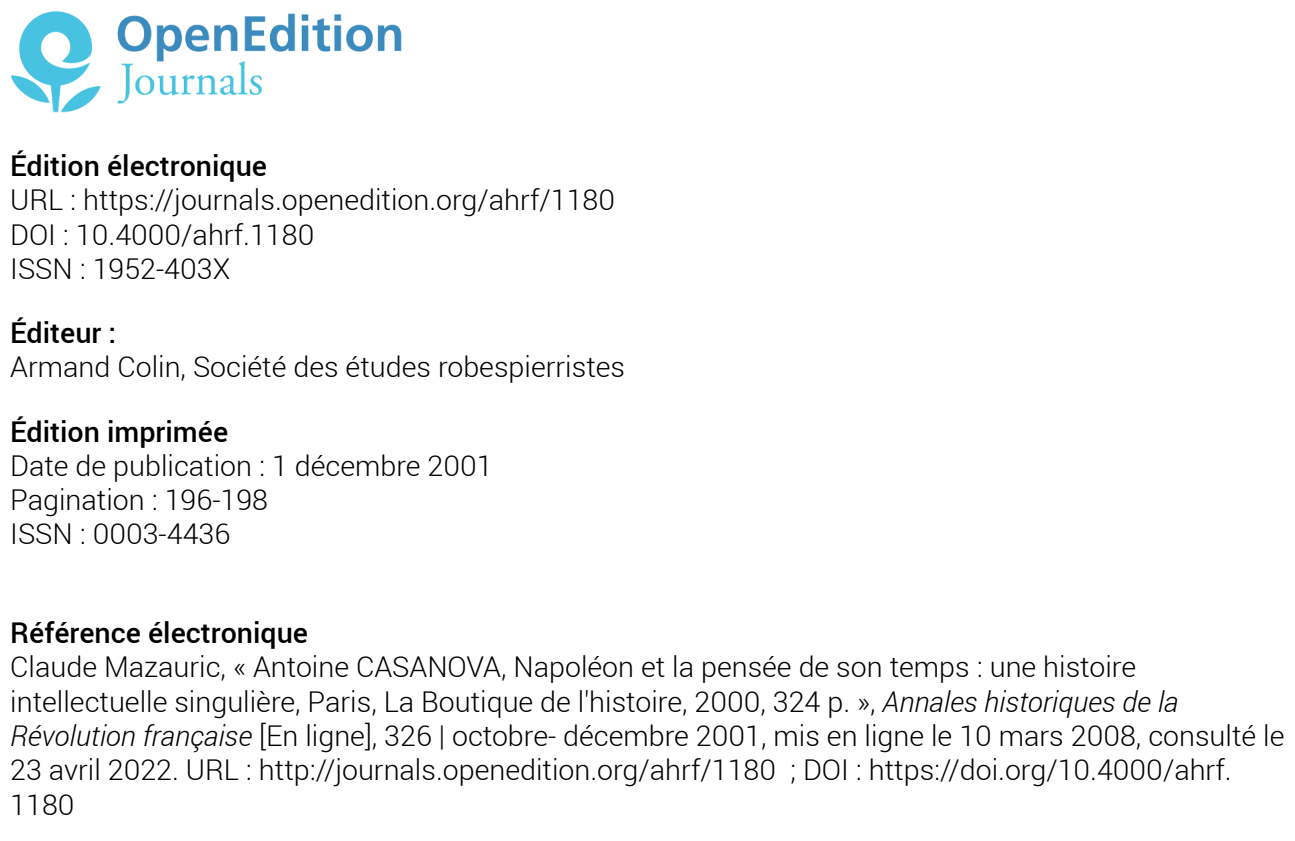

Ce document a été généré automatiquement le 23 avril 2022.

Tous droits réservés 


\title{
Antoine CASANOVA, Napoléon et la pensée de son temps : une histoire intellectuelle singulière, Paris, La Boutique de l'histoire, 2000, $324 \mathrm{p}$.
}

\author{
Claude Mazauric
}

On le sait par ses grands travaux d'érudition et de recherches en histoire et anthropologie, A. Casanova est non seulement un historien majeur de la Corse, auquel la revue Études corses a naguère rendu un hommage impressionnant, il est aussi l'un des plus pertinents parmi les analystes de la culture des Lumières, observées sur le versant de leur anthropologie. En consacrant un gros livre à Napoléon Bonaparte, penseur de son temps, penseur immergé dans son temps, Casanova s'efforce de comprendre l'homme d'action dans son individualité « singulière » par référence aux savoirs qu'il a reçus et à la manière dont s'est façonnée sa personnalité intellectuelle mais sans rien oblitérer, s'agissant des textes émanant de lui-même, des parades auto justificatives qu'il a produites in fine pour aider à construire ce mausolée de gloire dont il ne doutait pas que la postérité le consacrerait à sa mémoire. Dans ce nouveau livre sur Napoléon, on cherchera en vain une nouvelle biographie ou une apologie facile. Si l'« Empereur » $\mathrm{y}$ est toujours présent - avec le « $\mathrm{E} »$ majuscule qui plaît aux admirateurs ! - il ne l'est en réalité qu'à travers les "élaborations intellectuelles» qui accompagnent, fondent, illustrent et justifient le cas échéant, l'orientation de sa politique et les grands choix qui s'imposèrent à lui ou qu'il imposa à la Corse puis à la République française et enfin à l'Europe pendant une durée qui fut celle d'une génération entière. Ne cherchons donc pas dans ce livre une nouvelle glose à mettre au compte de la napoléophilie - quoique celle-ci ne soit pas tout à fait étrangère à la plume de l'auteur! - mais enregistrons comme un effort très réussi, l'enquête tout à fait originale, soigneuse et argumentée entreprise par A. Casanova dans ce qui constitua l'univers mental, intellectuel et conceptuel (mais non pas affectif), d'un général républicain devenu Premier consul puis Empereur des Français. 
2 Se lancer dans cette entreprise exigeait de l'audace et surtout de la patience. Le corpus de l'œuvre napoléonienne, on le sait, est immense, divers, problématique... et la bibliographie comme la liste des travaux à l'homme Napoléon consacrés, décourage l'ambition d'exhaustivité ! Fort sagement, l'auteur a pris le parti d'un retour critique et comparatif aux sources elles-mêmes : le Mémorial évidemment, le Journal de O'Meara Barry (Napoléon dans l'exil), le Journal de Sainte-Hélène de Gourgaud, les Récits de Montholon, les Mémoires de Marchand, les Cahiers de Sainte-Hélène de Bertrand, tous les écrits antérieurs de Napoléon Bonaparte, ouvrages comme le Souper de Beaucaire, discours, notes, ordres du jour, textes officiels, correspondances, témoignages de conversations ou d'audiences, etc., eux-mêmes resitués quand le propos le permet, dans l'utilisation qu'en fit le légendaire du XIX siècle (cf. Natalie PETITEAU, Napoléon de la mythologie à l'histoire, Paris, 1995). De cette puissante compilation dans laquelle l'auteur aurait pu se noyer, sort par la magie d'une confrontation ordonnée, une synthèse intelligente, à la fois neuve et percutante.

3 La première partie du livre est consacrée à l'étude des fondements philosophiques de l'anthropologie napoléonienne: un athéisme essentiel fondé sur une conception « matérialiste » du monde de type holbachien; la matière serait étendue et mouvement et c'est dans la nature, c'est-à-dire par elle que, fluides, éther et électricité se combinant, se serait construite la matière vivante. Cela autorise-t-il à évoquer le recours chez Napoléon à une catégorie générale de pensée critique qu'on tiendrait pour un "matérialisme» anticipateur de type contemporain? J'avoue n'en avoir pas été entièrement convaincu et certains verront peut-être ici une reconstruction téléologique quoique, en regardant de près les textes cités, on soit incontestablement enclin à penser que Napoléon a porté à leurs conséquences ultimes les esquisses d'une critique matérialiste inspirée des encyclopédistes et de Diderot ou inscrite dans la tradition condillacienne.

4 Une très large seconde partie est consacrée à Napoléon comme penseur de l'histoire ; on le savait attentif et passionné par l'histoire saisie dans la longue durée des civilisations (le christianisme, l'islam) mais on le voit aussi préoccupé de comprendre les crises comme événements spécifiques : la collecte des citations est ici exemplaire et montre la précocité, la continuité et la diversité de genre, des préoccupations de l'Empereur en ce domaine. C'est ici que l'on trouvera matière à se représenter la manière dont Napoléon conçoit la Révolution française dont il se tient pour l'héritier, quoique en partie seulement: nécessité de la Révolution au regard des besoins nouveaux de l'économie et de la société autant que des mœurs, émergence et puissance de la référence nationale, risque de décomposition de l'effort prométhéen sous l'effet des impatiences sociales et des petitesses humaines, vaste affrontement de l'ancien et du nouveau dont la guerre européenne de 1792 à 1815 - malgré la courte paix hélas précaire de 1802-1803 qui n'en a pas vraiment interrompu le cours - a fixé les enjeux sur un terrain qui n'était peut-être pas celui qui avait la préférence de Napoléon nous dira celui-ci au terme de sa vie. Mais celui qui détruisit la République - on y revient p. 222 et suivantes - ne cessa de se vouloir fidèle à la Révolution et aux intérêts de la "classe moyenne ", accoucheuse de "progrès ». Fidélité ? La troisième partie de l'essai, à partir d'une interrogation sur le silence de l'Empereur à propos de Thermidor, compose une étrange mais convaincante analyse du philo-robespierrisme de l'exilé de Sainte-Hélène ; entre ce « Robespierre, miroir de Bonaparte » (p.158) et le chef dont la réflexion ultime porte sur l'angoisse de l'homme d'État face à la décision et au sens de 
son action, comme Jean Massin autrefois et à la suite de beaucoup d'autres, Antoine Casanova voit une certaine forme de continuité et beaucoup de familiarité: protagonistes majeurs de l'histoire française de leur temps, le cousinage est entre eux objectif. Mais il y ajoute une fine observation sur le remaniement de l'outillage conceptuel - on pourrait parler de bricolage - de l'Empereur entre le début et la fin d'une trajectoire qui le conduit d'un déterminisme de type mécaniste à un néodéterminisme faisant la part belle au hasard et à la probabilité : un effet peut-être des préoccupations systémiques de l'époque! La quatrième partie est consacrée tout entière à une réflexion critique sur les «constructions" autojustificatrices ou autocritiques et aux élaborations rétrospectives du reclus de Sainte-Hélène dans le contexte que chacun connaît: Napoléon s'y donne le rôle de l'irréductible opposant à l'Europe de la contre-révolution; il se fabrique l'image du plébéien volontaire, il y dresse le portrait de l'homme des réussites éclatantes comme celui des occasions manquées, la figure du héros qui refuse de sombrer dans la déréliction et de se soumettre au suicide vers lequel on le pousse, affichant un volontarisme théâtral et malgré tout humble, destiné à préparer, par ce qu'on dira de lui, le futur de la civilisation...

5 Livre important que l'essai d'Antoine Casanova! Il nous donne à voir dans une confrontation systématique de textes de diverses époques, un acteur majeur du mouvement historique pris dans sa subjectivité et l'évolution de ses représentations intellectuelles à partir du bagage conceptuel de savoirs divers qui furent ceux de son temps et du système de formation dont le hasard de la naissance le fit bénéficier. On y voit que Napoléon Bonaparte a peu fréquenté les Lumières anglaises, quasiment ignoré ce qui se pensait en Allemagne, de Leibniz à Kant ou Fichte, mais il était fort mathématicien et bon connaisseur de Montesquieu, Vico, Buffon, etc. Regrettons quand même que ce bel essai ne soit pas exempt, par tel ou tel détail, d'être tenu pour une contribution, même involontaire, à l'apologétique napoléonienne, ignorante des milliers de morts, de victimes et de sacrifiés de la tyrannie impériale, faisant bon marché de la liquidation ou de la perversion plébiscitaire des idéaux démocratiques contenus dans l'idée républicaine de 1792 à 1800, tenant pour négligeable le rétablissement de l'esclavage aux colonies et le retour contre-révolutionnaire du statut colonial lui-même. Certains lecteurs pourront aussi penser que Casanova "tire " Naoléon du côté d'une contemporanéité excessive... mais n'en fait-on pas autant depuis des décennies avec Chateaubriand, Guizot, Tocqueville, fournisseur des tartes à la crème libéralo-énarchiques? Le lecteur attentif et puriste pourra regretter le recours fréquent à des clichés lexicaux comme le terme "élaborations » (au pluriel toujours ou presque) pour «idée», le goût de l'auteur pour le vocabulaire psychologicoanthropologique quand le vocabulaire commun suffirait, l'oubli ou la sous-estimation de quelques grands travaux récents notamment sur la période du Directoire, mais cela n'est rien au regard de l'originalité de la démarche analytique majeure de cette somme qui fait de Napoléon, d'abord un penseur majeur de son temps, au-delà du grand protagoniste politique et militaire de la nouvelle Europe des nations qui se construisit dans le siècle dont il inaugura la venue. 